\title{
Evaluation of a Cognitive Rehabilitation Protocol in HIV Patients with Associated Neurocognitive Disorders: Efficacy and Stability Over Time
}

\author{
Alessandro Livelli ${ }^{1,2}$, Gian Carlo Orofino ${ }^{1}$, Andrea Calcagno ${ }^{3}$, Mariana Farenga ${ }^{1}$, \\ Donatella Penoncelli ${ }^{1}$, Marta Guastavigna ${ }^{1}$, Sinibaldo Carosella ${ }^{1}$, Pietro Caramello ${ }^{1}$ and \\ Lorenzo Pia ${ }^{2 *}$ \\ ${ }^{1}$ Division A of Infectious Diseases, Amedeo of Savoia Hospital, Torino, Italy, ${ }^{2}$ SpAtial, Motor and Bodily Awareness Research \\ Group, Department of Psychology, University of Torino, Torino, Italy, ${ }^{3}$ Unit of Infectious Diseases, Department of Medical \\ Sciences, University of Torino, Torino, Italy
}

OPEN ACCESS

Edited by:

Nuno Sousa,

University of Minho, Portugal

Reviewed by:

Satoru Otani,

Ryotokuji University, Japan

Jan Egil Nordvik,

Sunnaas Rehabilitation Hospital,

Norway

${ }^{*}$ Correspondence:

Lorenzo Pia

lorenzo.pia@unito.it

Received: 25 April 2015 Accepted: 30 October 2015 Published: 16 November 2015

Citation:

Livelli A, Orofino GC, Calcagno A, Farenga $M$, Penoncelli $D$,

Guastavigna M, Carosella $S$

Caramello P and Pia L (2015) Evaluation of a Cognitive Rehabilitation

Protocol in HIV Patients with Associated Neurocognitive Disorders: Efficacy and Stability Over Time. Front. Behav. Neurosci. 9:306. doi: 10.3389/fnbeh.2015.00306
The primary aim of the present study was to evaluate the efficacy and stability over time of a cognitive rehabilitation protocol (restorative and compensatory approach) in HIV/AIDS patients with HIV-associated Neurocognitive Disorder (HAND). At baseline, 32 HIV/AIDS patients (16 with and 16 without HAND) were assessed with a neuropsychological battery (i.e., pre-assessment) consisting of 22 tests covering eight cognitive domains. Then, the experimental group was administered over 4 months a cognitive rehabilitation protocol aimed at improving four cognitive domains by means of eight paper and pencil/computer-based exercises. The control group received guideline-adherent clinical care (i.e., standard of care). At the end of the cognitive treatment, both groups were re-administered the neuropsychological battery (i.e., post-assessment). Additionally, 6 months after post-assessment, the experimental group was given the same neuropsychological battery (i.e., follow up-assessment). In order to test the efficacy of the cognitive rehabilitation protocol, we compared between groups the results of the neuropsychological battery at the pre- and post-assessments. In order to evaluate the stability over time, the effects of the cognitive rehabilitation protocol was examined comparing within the experimental group the results of the neuropsychological battery at post- and follow up-assessments. Our results show that the two groups did not differ at the pre-assessment, but differed at post-assessment. Specifically, the experimental group showed a significant improvement in five domains (Learning and memory, Abstraction/executive functioning, Verbal fluency, Attention/working memory, and Functional), whereas the control group significantly worsened in the same domains. The improvement of the experimental group did not change in the follow up-assessment in two domains (Abstraction/executive functioning, Attention/working memory, and Functional). Overall, these findings support the efficacy and, to some extent, the stability over time of our cognitive rehabilitation protocol.

Keywords: HIV, cognitive impairment, HIV-associated neurocognitive disorders, neurocognitive rehabilitation, HAND 


\section{INTRODUCTION}

Combination antiretroviral therapy has extended the survival of patients living with Human Immunodeficiency Virus (HIV) and, as a result, HIV is becoming a chronic disease. At the same time, however, patients appear to be more commonly affected by physical, social and cognitive disabilities (e.g., O'Brien et al., 2014) than the general population. For instance, in a populationbased sample of people living with HIV, over $80 \%$ of patients reported at least one impairment, activity limitation or social participation restriction (Rusch et al., 2004). One of the most common causes of disability is HIV-associated Neurocognitive Disorder (HAND; Heaton et al., 2010). The largest HAND study to date reported deficits in 52\% of HIV-seropositive adults (Heaton et al., 2010). Since, these disabilities can affect clinical outcomes, cognitive rehabilitation may be a key step in the maintenance or improvement of quality of life of HIV patients.

Consensus research criteria for classifying HAND were published in 2007 (Antinori et al., 2007). Specifically, the authors proposed three categories: Asymptomatic Neurocognitive Impairment (ANI), Mild Neurocognitive Disorder (MND), and HIV-Associated Dementia (HAD). The taxonomy is based on objective diagnostic criteria and account for daily functioning. The diagnosis of mild (MND) or severe (HAD) symptomatic HAND, for instance, requires a functional decline in at least two cognitive domains.

While the incidence of HAD has been reduced with combination antiretroviral therapy, the prevalence of less severe forms of neurocognitive disorders (i.e., ANI and MND) has remained relatively stable. Further, a large longitudinal study found that both ANI and MND were associated with cognitive worsening (Grant et al., 2014). One explanation for the persistence of mild HAND is antiretroviral neurotoxicity (Giunta et al., 2011; Robertson et al., 2012). Such toxicity may occur via direct drug effects on neurons and glia or indirectly via druglinked effects on other organs, such as the cardiovascular system (Underwood et al., 2015).

The development of treatments for HAND is an important unmet clinical need. With regard to cognitive rehabilitation, three studies have been published to date (Boivin et al., 2010; Becker et al., 2012; Vance et al., 2012) but none employed HAND diagnoses as inclusion criteria and all used the so-called restorative approach that aims to restore the neural circuits underlying impaired cognitive processes by means of practice and focused training exercises. These studies reported positive effects on visual learning (Boivin et al., 2010) and speed of information processing (Boivin et al., 2010; Vance et al., 2012). However, the improvement did not extend to other ecologically relevant cognitive domains, such as executive functioning or working memory, and the durability of the benefits was not strongly addressed.

In the present study, we aimed to examine the efficacy and durability of a cognitive rehabilitation treatment for HAND in HIV+ adults taking suppressive antiretroviral therapy. To maximize the ecological impact of the training, our intervention combined the restorative approach of prior studies with the compensatory approach known to better enable learning of new strategies and minimizing the impact of remaining impairment (Robertson and Murre, 1999; Cicerone et al., 2005; DamsO'Connor et al., 2009). We predicted a significant improvement of the neuropsychological picture in HAND-treated, respect to HAND-untreated patients. Additionally, we predicted the stability over time of these effects.

\section{MATERIALS AND METHODS}

\section{Participants}

Thirty-two patients (16 with and 16 without HAND) in care to the Infectious Disease Unit "Division A," Amedeo of Savoia Hospital (Turin, Italy) provided written informed consent to participate in the study, which was approved by the Local Bioethics Committee (ASL TO2). The study did not include any pharmacological intervention, and it was performed in accordance with the ethical standards described in the Declaration of Helsinki (World Medical Association, 2013).

Patients were randomized 1:1 to either the experimental group or the control group. The two groups did not differ in demographic, clinical, or treatment characteristics (all $p>0.05$, see Table 1).

Both groups were administered a pre-assessment neuropsychological battery (see below). Then, the experimental group was given the cognitive rehabilitation intervention for 4 months (see below for the full description) and the control group was given standard of care. At the end of the cognitive treatment, both groups were re-administered (post-assessment) the neuropsychological battery. The experimental group was also given the neuropsychological battery 6 months after the post-assessment (follow up) in a within-subjects randomized order. Raw scores on each

TABLE 1 | Demographical/Clinical data of the two groups of patients and their statistical comparisons at baseline (pre-assessment).

\begin{tabular}{lccc}
\hline & $\begin{array}{c}\text { Experimental } \\
\text { group }\end{array}$ & $\begin{array}{c}\text { Control } \\
\text { group }\end{array}$ & $\boldsymbol{p}$ \\
\hline Sample size & 16 & 16 & $>0.05$ \\
Plasma HIV-RNA $^{\mathrm{b}}(\leq 50 \mathrm{c} / \mathrm{mL})^{2}$ & 16 & 16 & $>0.05$ \\
Years HIV infected $^{\mathrm{a}}$ & $11.25(5.8)$ & $8.75(6)$ & $>0.05$ \\
Current CD4+ T-cell count $^{\mathrm{c}}$ & $539(299-611)$ & $614(369-810)$ & $>0.05$ \\
Nadir CD4 T-cell count $^{\mathrm{C}}$ & $212(100-273)$ & $177(57-265)$ & $>0.05$ \\
HCV seropositive $^{\mathrm{b}}(\%)$ & $3(19)$ & $1(6)$ & $>0.05$ \\
Number of current antiretrovirals $^{\mathrm{C}}$ & $3(3-4)$ & $3(3-4)$ & $>0.05$ \\
CPE (CNS penetration $^{\text {effectiveness score) of current }}$ & $7.1(2)$ & $7.5(1.3)$ & $>0.05$ \\
regimen $^{\mathrm{a}}$ & & & \\
Age (years) $^{\mathrm{C}}$ & $47.5(12.2)$ & $50(8.4)$ & $>0.05$ \\
Ethnicity (Cau) $^{\mathrm{b}}$ & $16(100)$ & $14(87.5)$ & $>0.05$ \\
Education $^{\mathrm{c}}$ & $10(3)$ & $9(3.9)$ & $>0.05$ \\
Gender (Women) $^{\mathrm{b}}$ & $5(31)$ & $3(19)$ & $>0.05$ \\
\hline
\end{tabular}

Mean (SD) Median (IQR) or Number (\%).

${ }^{a}$ Mean (Standard deviation).

${ }^{b}$ Number (\%).

${ }^{c}$ Median (Inter-quartile range). 
TABLE 2 | Groups' pre-, post-, and follow up- raw/T-scores on each test/domain of the neuropsychological battery.

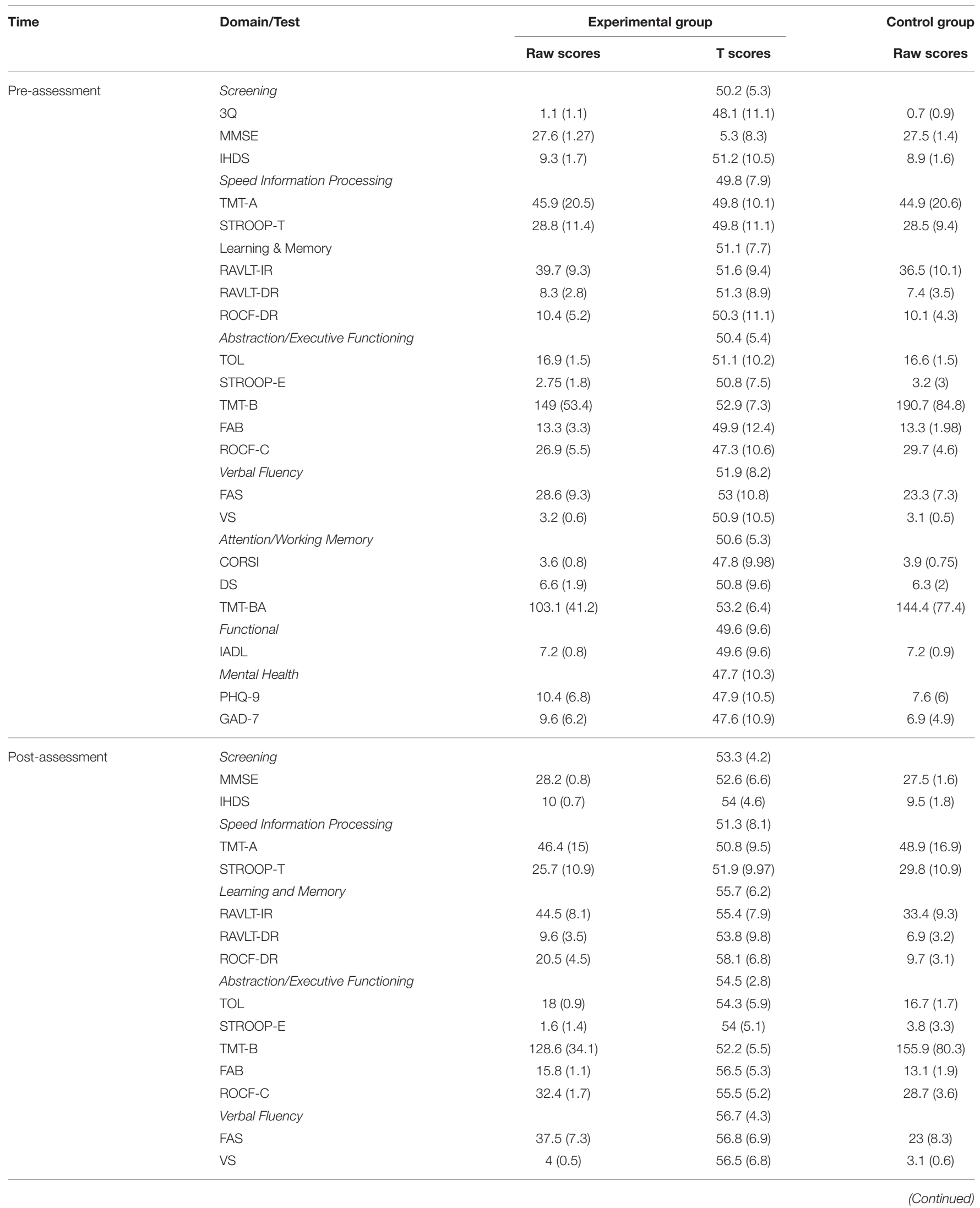


TABLE 2 | Continued

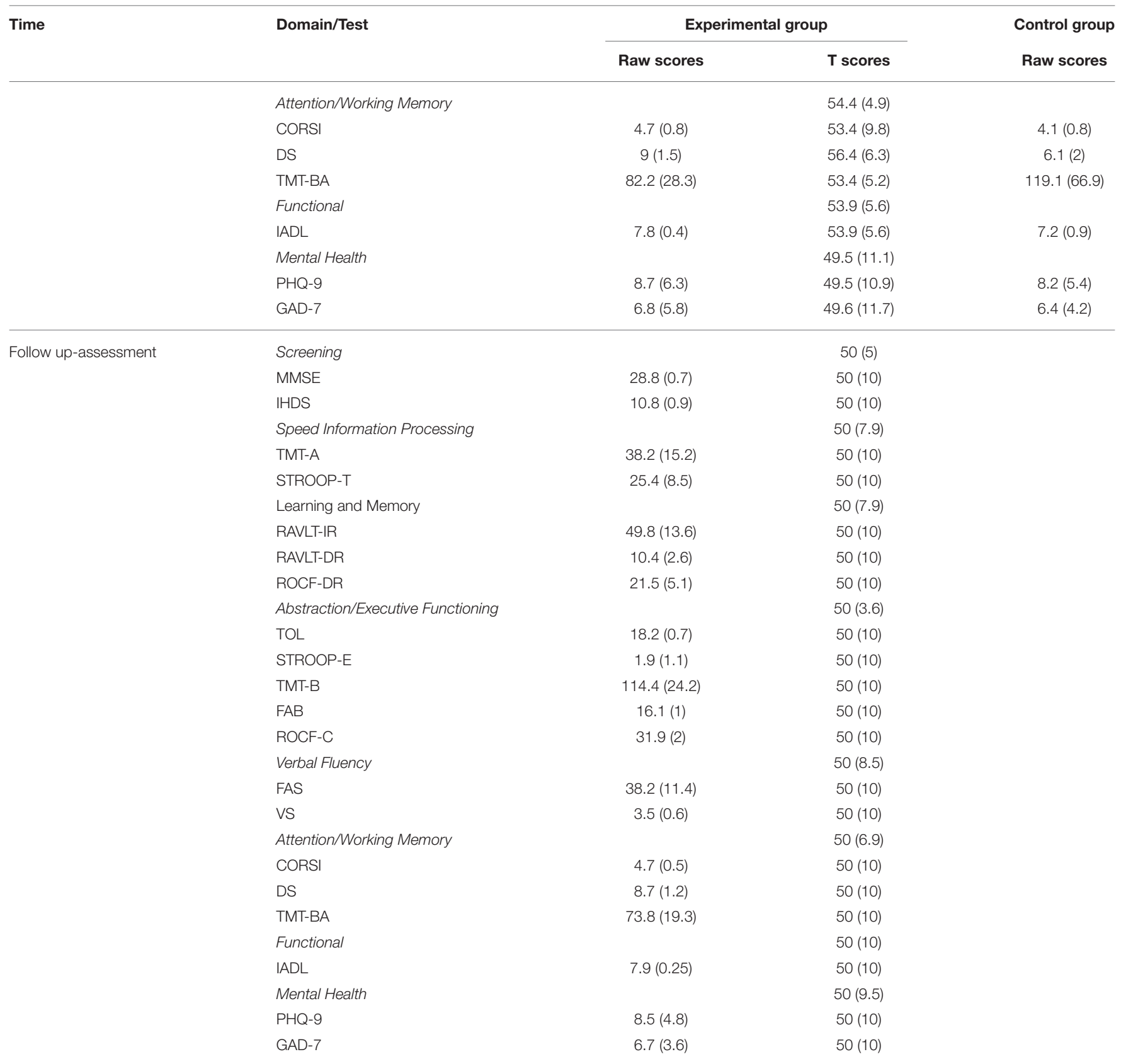

Scores are corrected for age, educational level and gender in the Italian population.

3Q, Simioni's 3 question test, <1 pathological; MMSE, Mini Mental State Examination, <26 pathological; IHDS, International HIV Dementia Scale, <10 pathological; TMT-A, Trial Making Test Part A, >68 pathological; STROOP-T, Stroop Time, $\geq 31.66$ pathological; RAVLT-IR, Rey Auditory Verbal Learning Test Immediate Recall, <32.26 pathological; RAVLT-DR, Rey Auditory Verbal Learning Test Delayed Recall, $<5.8$ pathological; ROCF-DR, Rey-Osterrieth Complex Figure Delayed Recall, $\leq 11.22$ pathological; TOL, Tower of London, <15 pathological; STROOP-E, Stroop Errors, $\geq 2.81$; TMT-B, Trial Making Test Part B, >177 pathological; FAB, Frontal Assessment Battery, <14.4 pathological; ROCF-C, Rey-Osterrieth Complex Figure Copy, $\leq 30.04$ pathological; FAS, Phonemic Fluency; <21.33 pathological; VS, Verbal Span, <3.5 pathological; CORSI, Corsi block-tapping test, <3.76 pathological; DS, Digit Span, <8 pathological; TMT-BA, Trial Making Test Part BA, > 111 pathological; IADL, Instrumental Activity of Daily Living Questionnaire, $\leq 6$ pathological; PHQ-9, Patient Health Questionnaire, > 15 pathological; GAD-7, Generalized Anxiety Disorder, >15 pathological.

Mean (Standard error).

test are reported in Table 2. Scores were corrected for age, educational level and gender using Italian normative data. Since the Grooved Pegboard Test has no standardization, it is not reported.

\section{Neuropsychological Battery}

Initially, 220 consecutive HIV/AIDS patients were administered three different screening tests of the neuropsychological battery in a between-subjects randomized order: 
- Mini Mental State Examination (MMSE; Folstein et al., 1975)

- International HIV Dementia Scale (IHDS; Sacktor et al., 2005)

- Simioni’s 3 question test (3Q; Simioni et al., 2010)

Then, patients with a score below the cut off in MMSE, and/or at three questions test, and/or at the IHDS were administered in a between-subjects randomized order the rest of the neuropsychological battery $(N=110)$ composed by 19 tests covering seven different cognitive domains:

- Speed of information processing

- Trail Making Test Part A (TMT-A, Giovagnoli et al., 1996)

- Stroop Color Test-Time (STROOP-T, Caffarra et al., 2002a)

- Learning and Memory

- Rey Auditory Verbal Learning Test Immediate Recall (RAVLT-IR, Carlesimo et al., 1996)

- Rey Auditory Verbal Learning Test Delayed Recall (RAVLTIR, Carlesimo et al., 1996)

- Rey-Osterrieth Complex Figure Delayed Recall (ROCF-DR, Caffarra et al., 2002b)

\section{- Abstraction/Executive Functioning}

- Tower of London simplified version (ToL; Allamanno et al., 1987)

- Stroop Color Test-Errors (STROOP-E, Caffarra et al., 2002a)

- Trail Making Test Part B (TMT-B, Giovagnoli et al., 1996)

- Frontal Assessment Battery (FAB, Appollonio et al., 2005)

- Rey-Osterrieth complex Figure Copy (ROCF-C, Caffarra et al., 2002b)

- Verbal Fluency

- Phonemic Fluency (FAS, Carlesimo et al., 1995)

- Verbal Span (VS, Spinnler and Tognoni, 1987)

- Attention/Working Memory

- Corsi's block-tapping Test (CORSI, Orsini et al., 1987)

- Digit Span (DS, Orsini and Laicardi, 1997)

- Trail Making Test Part BA (TMT-BA, Giovagnoli et al., 1996)

- Motor

- Grooved Pegboard Test Dominant and non- dominant hands (Heaton et al., 1991)

- Functional

- Instrumental Activity of Daily Living Questionnaire (IADL, Lawton and Brody, 1969). This latter test was administered to investigate 10 areas of autonomy in activities of daily living: ability to use the phone, grocery shopping, preparing meals, take care of the house, laundry, moving away from home, assumption drugs, use of money, work ability, work efficiency.

\section{- Mental Health}

- Patient Health Questionnaire (PHQ-9, Kroenke and Spitzer, 2002)

- Generalized Anxiety Disorder (GAD-7, Kroenke and Spitzer, 2002)

Sixty of 110 tested patients (54\%) were diagnosed with HAND (48 ANI, $10 \mathrm{MND}$, and $2 \mathrm{HAD})$. Participants $(N=32)$ were selected among those with HAND based on specific eligibility criteria: receiving antiretroviral therapy for at least 6 months, plasma HIV RNA below 50 copies/mL for at least 6 months, CD4+ T lymphocyte count above 350 cells/ $\mu \mathrm{L}$ from at least 6 months, fluent Italian speaker, and absence of severe comorbidities, including neurodegenerative or psychiatric disease, metabolic encephalopathy, psychoactive drug use, alcohol use, or head trauma.

\section{Cognitive Rehabilitation Treatment}

The cognitive rehabilitation protocol included both paperand-pencil and computer-based exercises and it was composed of eight different exercises repeated in 36 sessions (around $50 \mathrm{~min}$ each) over 4 months. The eight tests were administered in a randomized-within subjects order. The protocol aimed at improving with the different group of tests four cognitive domains:

\section{- Attention}

- Time Pressure Management (Fasotti et al., 2000)

- Five minute paper-and-pencil exercise to improve functional impairments related to slowed information processing and complex attention. Patients learn compensatory strategies as, for instance, allowing sufficient time to manage a task

- Attention Process Training Task (Cicerone et al., 2005)

- Five minute paper-and-pencil exercise organized hierarchically to improve different components of attention: sustained, selective, alternating and divided attention. Patients learn compensatory strategies as, for instance, removing environmental distractors or employing using cues to maintain attention

- Visual-verbal memory and learning

- COG.I.T.O. (open platform. ASPHI and San Camillo Hospital, Turin

- Ten minute computer-based exercise to improve attention/visual-spatial memory. Patients have to encode daily-use objects in domestic environments (i.e., kitchen, bedroom, garage). Then, objects disappear and they have to put them back in their correct location, or recognizing them among distractors and relocating them, or writing their names and relocating them. From session to session, the available time decreases whereas the number of objects increases. 
○ Errorless Learning (Ehlhardt et al., 2005)

- Five minute paper-and-pencil exercise providing sufficient cues during training so that patients can only give correct responses. Then, cues are progressively sequentially reduced

- Process-Oriented Memory Learning (Huildebrandt et al., 2006)

- Five minute paper-and-pencil exercise to improve strategies adapted to different situations with memory requirements (e.g., practice, managing interferences between acquisition and recall, principles to optimize memory performance)

\section{- Executive Functioning and Working Memory}

- Metacognitive Strategy Training (Kennedy et al., 2008)

- Five minute paper-and-pencil exercise to improve daily problem-solving abilities (e.g., use of metacognitive approaches incorporating emotional self-regulation strategies which facilitates clear thinking)

- Goal Management Training (Levine et al., 2000)

- Five minute paper-and-pencil exercise to improve the ability to stop and think about what one is doing, identifying a specific goal, delineating the steps or achieve a goal and evaluating the outcomes

- Metacognitive Awareness

- Increased Awareness (10 min)

- One minute at the beginning of each session, 1 min at the end of each exercise for $10 \mathrm{~min}$ to improve awareness of neurocognitive deficits (Dams-O'Connor and Gordon, 2010).

\section{RESULTS}

Raw corrected scores on each neuropsychological test at pre pre-, post-, and follow up- were standardized (T-score). When necessary, the standard scores were reversed in order to keep the interpretation of all tests in the same direction, namely higher scores reflecting a better performance. Then the standard scores were averaged within each domain.

In order to evaluate the efficacy of the cognitive rehabilitation protocol, we performed a repeated measures ANOVA on each domain of the neuropsychological battery with the mean standard scores as dependent variables, TIME (two levels: pre-assessment, post-assessment) as within-subjects factor, and GROUP (two levels: experimental, control) as between-subjects factor.

Respect to "Screening," the TIME $\mathrm{x}$ group interaction resulted to be significant $\left[F_{(1,30)}=7.05, p=0.013\right]$. Post-hoc comparisons (Duncan) revealed that the two groups did not differ at pre-assessment (experimental group: mean $=50.23$, $S E=1.32$; control group: mean $=49.77, S E=1.31)$, but differed $(p=0.007)$ at post-assessment (experimental group: mean $=53.3, S E=1.74$; control group: mean $=46.7, S E=1.74)$; see Figure 1. The analysis on "Speed information processing" was not significant. As regards "Learning and memory," the TIME $\times$ GROUP interaction was significant $\left[F_{(1,30)}=31.58\right.$, $p<0.0001]$. Post-hoc comparisons (Duncan) revealed that the two groups did not differ at pre-assessment (experimental group: mean $=51.11, S E=1.96$; control group: mean $=48.89, S E=$ $1.95)$, but differed $(p=0.0002)$ at post-assessment (experimental group: mean $=55.75, S E=1.54$; control group: mean $=44.24$, $S E=1.54)$ because the mean score of the experimental group significantly $(p=0.0005)$ increased and the mean score of the control group significantly $(p=0.0001)$ decreased (see Figure 1). The TIME $\times$ GROUP interaction was significant $\left[F_{(1,30)}=21.42, p<0.0001\right.$ also for "Abstraction/Executive Functioning." Duncan post-hoc showed that the groups did not differ at pre-assessment (experimental group: mean $=50.41$, $S E=1.9$; control group: mean $=49.6, S E=1.38)$, but differed $(p<0.001)$ at post-assessment (experimental group: mean $=$ $54.51, S E=1.27$; control group: mean $=47.48, S E=1.27$ ) because the mean score of the experimental group significantly ( $p=0.003$ ) increased, and the mean score of the control group significantly ( $p=0.003$ ) decreased (see Figure 1). The same was true for the "verbal fluency" in which the TIME $\times$ GROUP interaction was significant $\left[F_{(1,30)}=11.45, p=\right.$ 0.002]. Duncan post-hoc analysis revealed that groups did not differ at pre-assessment (experimental group: mean $=51.95$, $S E=1.91$; control group: mean $=48.05, S E=1.91)$, but
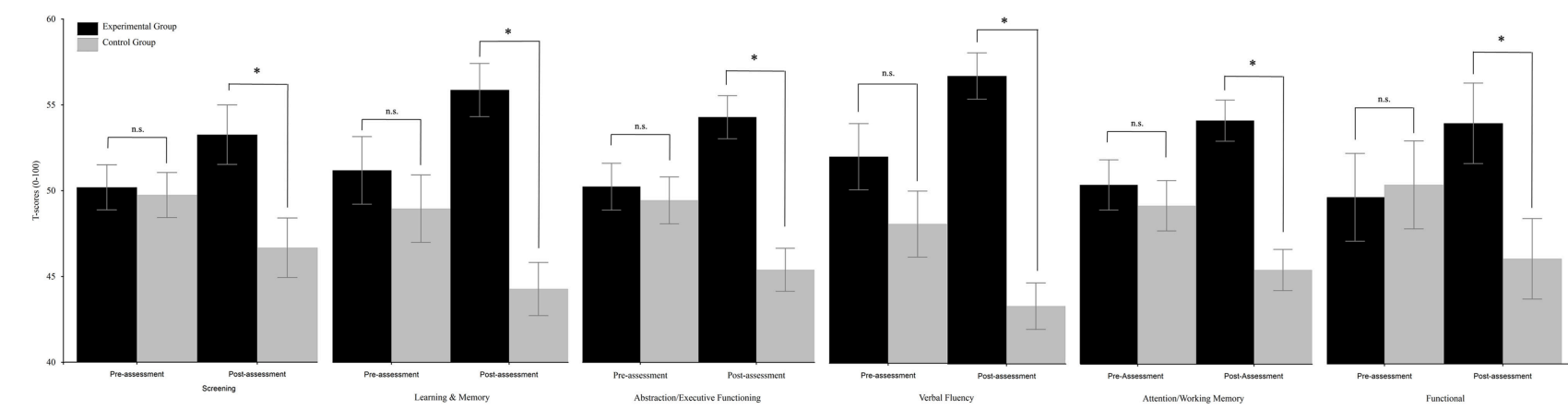

FIGURE 1 | Between-groups comparisons (domains) along time (pre- vs. post-assessment). Error bars represent standard errors. Asterisks indicate significant comparisons, n.s. indicate non-significant comparisons. 
differed $(p<0.0001)$ at post-assessment (experimental group: mean $=56.66, S E=1.34$; control group: mean $=43.34$, $S E=1.34)$ because the mean score of the experimental group significantly $(p=0.02)$ increased and the mean score of the control group significantly $(p=0.02)$ decreased (see Figure 1). The TIME $\times$ GROUP interaction was significant $\left[F_{(1,30)}=13.8, p=0.001\right]$ also for "Attention/Working memory." Duncan post-hoc analysis revealed that groups did not differ at pre-assessment (experimental group: mean $=50.62$, $S E=1.49$; control group: mean $=49.38, S E=1.49)$, but differed $(p=0.0001)$ at post-assessment (experimental group: mean $=54.42, S E=1.22$; control group: mean $=45.58$, $S E=1.22)$ because the mean score of the experimental group significantly $(p=0.016)$ increased and the mean score of the control group significantly $(p=0.016)$ decreased. The same was true for "Functional" in which the TIME $\times$ GROUP interaction was significant $\left[F_{(1,30)}=12.91, p=0.001\right]$. Duncan post-hoc revealed that groups did not differ at pre-assessment (experimental group: mean $=49.64, S E=2.54$; control group: mean $=50.36, S E=2.53)$, but differed $(p=0.042)$ at postassessment (experimental group: mean $=53.92, S E=2.31$; control group: mean $=46.08, S E=2.33$ ) because the mean score of the experimental group significantly $(p=0.021)$ increased and the mean score of the control group significantly $(p=0.021)$ decreased. The analysis on "Mental Health" was not significant.

In order to evaluate whether the treatment was able to induce permanent effects, we performed in the experimental group a within subject ANOVA on each domain of the neuropsychological battery which improved from pre- to post assessments. The mean standard scores were employed as dependent variables, and TIME (three levels: pre-assessment, post-assessment, follow up-assessment) as within-subjects factor. The analysis on "Learning and Memory" (see Figure 2) resulted to be significant $\left[F_{(2,30)}=8.92, p<0.001\right]$. Post-hoc comparisons (Duncan) revealed that the mean score significantly $(p=0.003)$ improved from pre- $($ mean $=51.1, S E=1.94)$ to post- $($ mean $=55.75, S E=1.55)$ assessment and worsened $(p<$ 0.001 ) from post- to follow up-assessment (mean $=50, S E=$ 2.1). Also the analysis on "Abstraction/executive functioning" (see Figure 2) was significant $\left[F_{(2,30)}=5, p=0.008\right]$ with the mean score significantly $(p=0.006)$ improved (Duncan) from pre- $($ mean $=50.4, S E=1.44)$ to post- $($ mean $=54.52$, $S E=0.7)$ assessment and worsened $(p=0.004)$ from postto follow up-assessment (mean $=50, S E=0.9$ ). The same was true (see Figure 2) for "Verbal Fluency" $\left[F_{(2,30)}=5.5\right.$, $p=0.01)$ with the mean score significantly $(p=0.03)$ improved (Duncan) from pre- $($ mean $=51.95, S E=2.06)$ to post- $($ mean $=56.67, S E=1.07)$ assessment and worsened $(p=0.005)$ from post- to follow up-assessment (mean = $50, S E=2.1$ ). As regards "Attention/working memory" (see Figure 2), the analysis resulted to be significant. However, post-hoc comparisons (Duncan) revealed that the mean score significantly $(p=0.03)$ improved from pre- $($ mean $=50.62, S E=$ $1.31)$ to post- $($ mean $=54.44, S E=1.22)$ assessment but did not change $(p>0.05)$ from post- to follow up-assessment (mean $=$ $50, S E=1.74$ ). The analysis on "Functional" (see Figure 2 ) was not significant $(p>0.05)$.

\section{DISCUSSION}

In the present study, we examined the efficacy of a cognitive rehabilitation protocol that included both restorative and

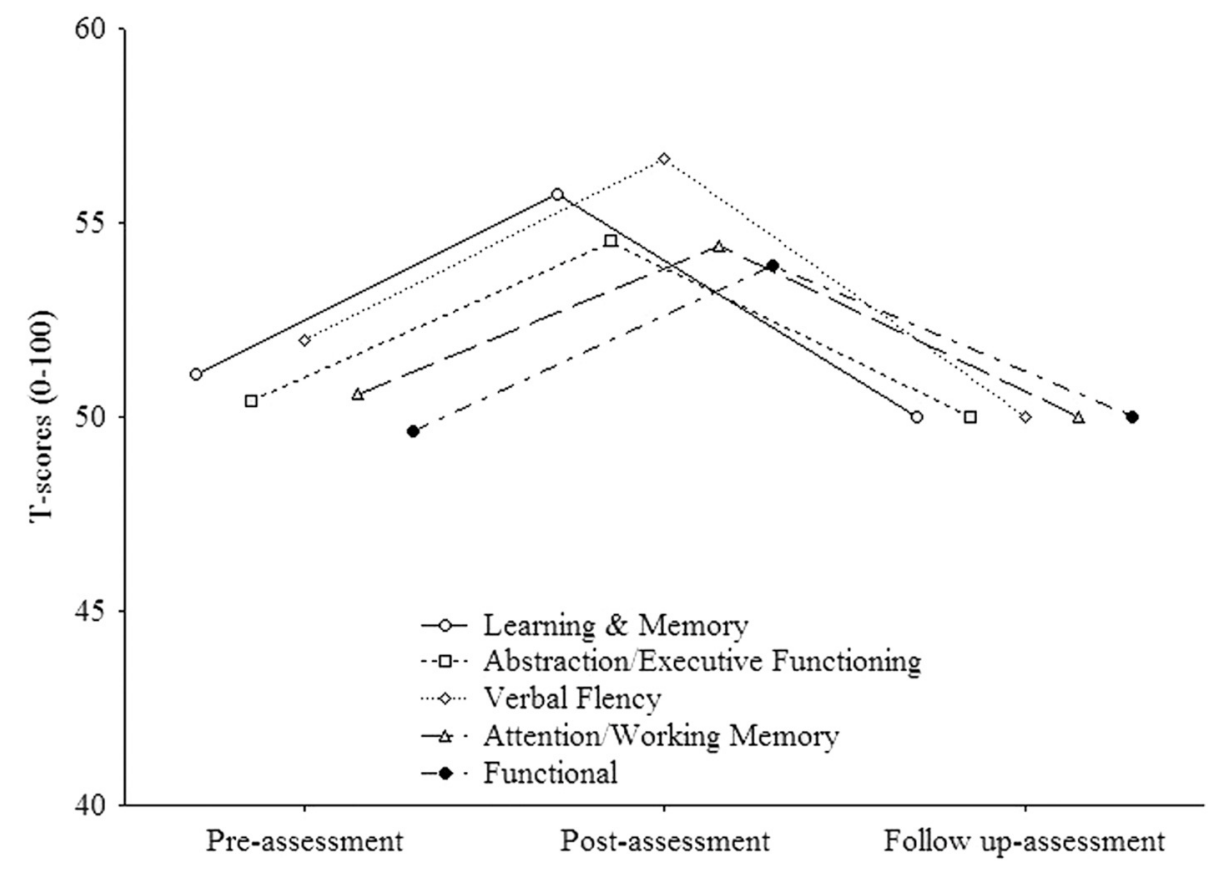

FIGURE 2 | Experimental group trends (improved domains) along time (post- vs. follow up-assessment. 
compensatory approaches for treatment of HAND. Our results show discordant clinical evolution in five out of eight domains in participants who received the intervention compared with those who did not: treated patients improved, untreated patients worsened. Additionally, the improvement proved to be stable over time in four of five domains.

Within the context of HIV, rehabilitation is a dynamic set of activities that can benefit the disease as well as linked social limitations and dysfunctions (Worthington et al., 2005). With the graying of the HIV population, the demand for rehabilitation services will likely increase in the near future (O'Brien et al., 2014). Since a substantial number of HIV patients have cognitive impairment (e.g., Heaton et al., 2010), expansion in rehabilitation services should include cognitive rehabilitation.

One definition of cognitive rehabilitation is a systematic, functionally oriented set of therapeutic activities. Cognitive improvement is achieved by both strengthening previously learned behavioral patterns and establishing new, compensatory ones (Bergquist and Malec, 1997). This is possible because of the brain plasticity, the changes of brain organization that subserve short- and long-term behavioral modifiability. These changes can be structural (i.e., remodeling of the brain's physical structures) or physiological (i.e., dynamic adjustment of cellular processes such as synapse formation that modulate conductance or resistance to impulse transmission; see, for instance, Berlucchi, 2011). These changes can include adaptation to novel environments, maturation, different learning types, and compensatory changes in response to functional loss (see, for instance, Berlucchi, 2011).

Successful cognitive rehabilitation treatment typically targets different cognitive abilities, such as attention, memory, perception, learning, and executive functioning. Metacognitive awareness, emotional regulation, social skills and community integration are other, important targets. The main aim of cognitive rehabilitation is to significantly reduce the impact of disease on daily living. More specific aims depend on the nature and severity of the individual cognitive, behavioral, physical, and emotional difficulties as well as on the specific individual premorbid achievements (Dams-O'Connor and Gordon, 2010).

With HAND, HIV mainly affects fronto-striatalthalamocortical circuitry, often being associated with decreased white matter volume (e.g., Thompson et al., 2005). As a consequence, cognitive deficits can cover a wide range of abilities. For instance, patients may develop impairments of visual/verbal working memory (Martin et al., 2001) as a consequence of central executive dysfunctions (Hinkin et al., 2002). Additionally, patients may show working memory impairments that can influence even higher-level cognitive abilities and daily activities (Martin et al., 2004). Nonetheless, HIV is frequently associated with difficulties of attentional processes (Hinkin et al., 2002), which, in turn, may negatively affect adherence to antiretroviral therapy (Levine et al., 2005, 2008). As a result, patients can experience multiple personal and professional difficulties and are frequently unemployed (Heaton et al., 1994; van Gorp et al., 2007).

In the present study, we aimed to maximize the effects of a cognitive rehabilitation intervention by training patients in both restorative (i.e., COG.I.T.O) and compensatory abilities (Time Pressure Management, Attention Process Training Task, Errorless Learning, Process-Oriented Memory Learning, Metacognitive Strategy Training, Goal Management Training, Increased Awareness). Each exercise was organized and administered in different steps with progressively increasing difficulty, suitable for gradual improvement of performance and for learning of any targeted ability. As written above, we found a significant improvement in the experimental, respect to the control, from pre- to post-assessments in Learning and memory, Abstraction/executive functioning, Verbal fluency, Attention/working memory, and Daily functioning. The improvement in Attention/working memory indirectly suggests benefits for daily functioning (working memory) and medication adherence (attention). The improvement in Learning and memory could explain the improvements in self-reported IADLs at post-assessment as well as the better work efficiency reported by treated patients. In contrast, the control group significantly worsened in the same domains.

At follow up-assessment, the cognitive improvement were stable (i.e., no significant difference respect to post-assessment) in two domains. It is worth noticing that previous long-term (i.e., 5 months to 1 year) benefits of cognitive rehabilitation (Tesar et al., 2005; Svendsen and Teasdale, 2006; Fink et al., 2010; Stuifbergen et al., 2012), have been related to patients continued use of learned strategies in ecological situations, as well as to the relevance of the intervention to the patient's daily functioning (Cicerone et al., 2000). Then, patients of experimental group used learned strategies in ecological contexts of daily living. However, in the other three domains, the experimental group's performance significantly worsened (and, indeed, this is also the overall trend). Broadly speaking, this is not surprising but, rather, consistent with the fact that most long-term follow up studies on the effects of acquired brain injuries show also some persisting effects (Klonoff et al., 1993; Dikmen et al., 2003; Wood and Rutterford, 2006). Additionally, ARV neurotoxicity and the potential HIV activities within the central nervous system acts against the cognitive rehabilitation process. Indeed, this might also explain, at least in part, the abovementioned fact that the control group worsened from pre- to post-assessment.

Our study had several limitations. First, the sample size is relatively small. Hence, further studies with larger samples are required to validate the findings. Secondly, and more importantly, the control group was administered standard of care only but not a structured activity, raising the possibility that repeating per se the cognitive rehabilitation protocol might have a role in the improvement observed in the experimental group. This possibility is countered by the fact that benefit was not observed in all domains, which would have been seen if the improvement was solely due to practice. Still, this remains a limitation of our investigation and future studies should employ active control activities such as generalized compensatory cognitive training or low cognitive demand computer activities (Weber et al., 2013). Interestingly, those studies might address the effects of different kind of interventions: restorative approaches vs. compensatory 
interventions, purely computer-based vs. purely paper-based. Beyond the presence of a control condition, such strategies would better enable proof of concept for the use of cognitive rehabilitation in HAND.

In conclusion, our intervention was associated with better cognitive performance. According to a recent call to action (Weber et al., 2013), future studies should prioritize development of specific cognitive rehabilitation interventions for HAND, particularly with emphasis on two issues. First, while our center is similar to other Italian centers, a specific battery of standardized tests for HIV populations should be developed in Italy to generalize our findings to different countries. Secondly, the impact of cognitive rehabilitation on daily living, quality of life, and medication adherence has to be clarified. Since unawareness is in general a significant barrier to treatment (Ownsworth et al.,

\section{REFERENCES}

Allamanno, N., Della Sala, S., Laiacona, M., Pasetti, C., and Spinnler, H. (1987). Problem solving ability in aging and dementia: normative data on a non-verbal test. Ital. J. Neurol. Sci. 8, 111-119. doi: 10.1007/BF02337583

Antinori, A., Arendt, G., Becker, J. T., Brew, B. J., Byrd, D. A., Cherner, M., et al. (2007). Updated research nosology for HIV-associated neurocognitive disorders. Neurology 69, 1789-1799. doi: 10.1212/01.WNL.0000287431. $88658.8 \mathrm{~b}$

Appollonio, I., Leone, M., Isella, V., Piamarta, F., Consoli, T., Villa, M. L., et al. (2005). The Frontal Assessment Battery (FAB): normative values in an Italian population sample. Neurol. Sci. 26, 108-116. doi: 10.1007/s10072-0050443-4

Becker, J. T., Dew, M. A., Aizenstein, H. J., Lopez, O. L., Morrow, L., Saxton, J., et al. (2012). A pilot study of the effects of internet based cognitive stimulation on neuropsychological function in HIV disease. Disabil. Rehabil. 34, 1848-1852. doi: $10.3109 / 09638288.2012 .667188$

Bergquist, T. F., and Malec, J. F. (1997). Psychology: current practice and training issues in treatment of cognitive dysfunction. Neurorehabilitation 8, 49-56. doi: 10.1016/S1053-8135(96)00208-9

Berlucchi, G. (2011). Brain plasticity and cognitive neurorehabilitation. Neuropsychol. Rehabil. 21, 570-578. doi: 10.1080/09602011.2011.573255

Boivin, M. J., Busman, R. A., Parikh, S. M., Bangirana, P., Page, C. F., Opoka, R. O., et al. (2010). A pilot study of the neuropsychological benefits of computerized cognitive rehabilitation in Ugandan children with HIV. Neuropsychology 24, 667-673. doi: 10.1037/a0019312

Caffarra, P., Vezzadini, G., Dieci, F., Zonato, F., and Venneri, A. (2002a). Una versione abbreviata del test di Stroop: dati normativi nella popolazione italiana. Rivista di Neurologia 4, 111-115.

Caffarra, P., Vezzadini, G., Dieci, F., Zonato, F., and Venneri, A. (2002b). ReyOsterrieth complex figure: normative values in an Italian population sample. Neurol. Sci. 22, 443-447. doi: 10.1007/s100720200003

Carlesimo, G. A., Caltagirone, C., and Gainotti, G. (1996). The Mental Deterioration Battery: normative data, diagnostic reliability and qualitative analyses of cognitive impairment. The Group for the Standardization of the Mental Deterioration Battery. Eur. Neurol. 36, 378-384. doi: $10.1159 / 000117297$

Carlesimo, G. A., Caltagirone, C., Gainotti, G., and Nocentini, U. (1995). Batteria per la valutazione del deterioramento mentale (parte 2): standardizzazione e affidabilità diagnostica nell'identificazione di pazienti affetti da sindrome demenziale. Arch. Psicol. Neurol. Psichiatr. 56, 471-488.

Cicerone, K. D., Dahlberg, C., Kalmar, K., Langenbahn, D. M., Malec, J. F., Bergquist, T. F., et al. (2000). Evidence-based cognitive rehabilitation: recommendations for clinical practice. Arch. Phys. Med. Rehabil. 81, 1596-1615. doi: 10.1053/apmr.2000.19240

Cicerone, K. D., Dahlberg, C., Malec, J. F., Langenbahn, D. M., Felicetti, T., Kneipp, S., et al. (2005). Evidence-based cognitive rehabilitation: updated review of the
2002) and since more than $50 \%$ of people with HIV have poor insight of their cognitive deficits (Weber et al., 2013), more specific measures and more sensitive interventions should be developed.

\section{ACKNOWLEDGMENTS}

The study has been funded by G. Goria Foundation Scholarship "Master dei Talenti della Società Civile" to AL, a Gilead, Abbvie, MSD, BMS, Janssen-Cilag, ViiV Healthcare research grant to GO, a Gilead and Bristol Meyer-Squib research grant to AC, a ICONA research grant to $\mathrm{MF}$ and a ICONA research grant to MG. A special thanks to Amedeo of Savoia Hospital, Division A and the whole Department of Infectious Diseases. We are grateful to Scott Letendre who carefully checked the English. literature from 1998 through 2002. Arch. Phys. Med. Rehabil. 86, 1681-1692. doi: 10.1016/j.apmr.2005.03.024

Dams-O'Connor, K., and Gordon, W. A. (2010). Role and impact of cognitive rehabilitation. Psychiatr. Clin. North Am. 33, 893-904. doi: 10.1016/j.psc.2010. 08.002

Dams-O'Connor, K., Lebowitz, M., Cantor, J., Gordon, W., and Spina, L. (2009). Feasibility of a computerized cognitive skill-building program in an inpatient TBI rehabilitation setting. Arch. Phys. Med. Rehabil. 90, e19-e20.

Dikmen, S. S., Machamer, J. E., Powell, J. M., and Temkin, N. R. (2003). Outcome 3 to 5 years after moderate to severe traumatic brain injury. Arch. Phys. Med. Rehabil. 84, 1449-1457. doi: 10.1016/S0003-9993(03)00287-9

Ehlhardt, L. A., Sohlberg, M. M., Glang, A., and Albin, R. (2005). TEACHM: a pilot study evaluating an instructional sequence for persons with impaired memory and executive functions. Brain Inj. 19, 569-583. doi: $10.1080 / 002699050400013550$

Fasotti, L., Kovacs, F., Eling, P. A., and Brouwer, W. H. (2000). Time pressure management as a compensatory strategy training after closed head injury. Neuropsychol. Rehabil. 10, 47-65. doi: 10.1080/096020100389291

Fink, F., Rischkau, E., Butt, M., Klein, J., Eling, P., and Hildebrandt, H. (2010). Efficacy of an executive function intervention programme in MS: a placebocontrolled and pseudo-randomized trial. Mult. Scler. 16, 1148-1151. doi: $10.1177 / 1352458510375440$

Folstein, M. F., Folstein, S. E., and McHugh, P. R. (1975). "Mini-mental state”. A practical method for grading the cognitive state of patients for the clinician. $J$. Psychiatr. Res. 12, 189-198. doi: 10.1016/0022-3956(75)90026-6

Giovagnoli, A. R., Del Pesce, M., Mascheroni, S., Simoncelli, M., Laiacona, M., and Capitani, E. (1996). Trail making test: normative values from 287 normal adult controls. Ital. J. Neurol. Sci. 17, 305-309. doi: 10.1007/BF01997792

Giunta, B., Ehrhart, J., Obregon, D. F., Lam, L., Le, L., Jin, J., et al. (2011). Antiretroviral medications disrupt microglial phagocytosis of $\beta$-amyloid and increase its production by neurons: implications for HIV-associated neurocognitive disorders. Brain 4:23. doi: 10.1186/1756-6606-4-23

Grant, I., Franklin, D. R. Jr., Deutsch, R., Woods, S. P., Vaida, F., Ellis, R. J., et al. (2014). Asymptomatic HIV-associated neurocognitive impairment increases risk for symptomatic decline. Neurology 82, 2055-2062. doi: 10.1212/WNL.0000000000000492

Heaton, R. K., Clifford, D. B., Franklin, D. R. Jr., Woods, S. P., Ake, C., Vaida, F., et al. (2010). HIV-associated neurocognitive disorders persist in the era of potent antiretroviral therapy: CHARTER study. Neurology 75, 2087-2096. doi: 10.1212/WNL.0b013e318200d727

Heaton, R. K., Grant, I., and Matthews, C. G. (1991). Comprehensive Norms for an Expanded Halstead-Reitan Battery: Demographic Corrections, Research Findings, and Clinical Applications. Odessa, FL: Psychological Assessment Resources, Inc.

Heaton, R. K., Velin, R. A., McCutchan, J. A., Gulevich, S. J., Atkinson, J. H., Wallace, M. R., et al. (1994). Neuropsychological impairment in human immunodeficiency virus-infection: implications for employment, HNRC 
Group. HIV Neurobehavioral Research Center. Psychosom. Med. 56, 8-17. doi: 10.1097/00006842-199401000-00001

Hinkin, C. H., Hardy, D. J., and Mason, K. I. (2002). Verbal and spatial working memory performance among HIV-infected adults. J. Int. Neuropsychol. Soc. 8, 532-538. doi: 10.1017/S1355617702814278

Huildebrandt, H., Bussmann-Mork, B., and Schwendermann, G. (2006). Group therapy for memory impaired patients: a partial remediation is possible. J. Neurol. 253, 512-519. doi: 10.1007/s00415-006-0013-6

Kennedy, M. R., Coelho, C., Turkstra, L., Ylvisaker, M., Moore Sohlberg, M., Yorkston, K., et al. (2008). Intervention for executive functions after traumatic brain injury: a systematic review, meta-analysis and clinical recommendations. Neuropsychol. Rehabil. 18, 257-299. doi: 10.1080/09602010701748644

Klonoff, H., Clark, C., and Klonoff, P. S. (1993). Long-term outcome of head injuries: a 23 year follow up study of children with head injuries. J. Neurol. Neurosurg. Psychiatr. 56, 410-415. doi: 10.1136/jnnp.56.4.410

Kroenke, K., and Spitzer, R. L. (2002). The PHQ-9: a new depression diagnostic and severity measure. Psychiatr. Ann. 32, 509-521. doi: 10.3928/0048-571320020901-06

Lawton, M. P., and Brody, E. M. (1969). Assessment of older people: selfmaintaining and instrumental activities of daily living. Gerontologist 9, 179-186. doi: 10.1093/geront/9.3_Part_1.179

Levine, A. J., Hinkin, C. H., Castellon, S. A., Mason, K. I., Lam, M. N., Perkins, A., et al. (2005). Variations in patterns of highly active antiretroviral therapy (HAART) adherence. AIDS Behav. 9, 355-362. doi: 10.1007/s10461-005-9009-y

Levine, A. J., Hardy, D. J., Barclay, T. R., Reinhard, M. J., Cole, M. M., and Hinkin, C. H. (2008). Elements of attention in HIV-infected adults: evaluation of an existing model. J. Clin. Exp. Neuropsychol. 30, 53-62. doi: $10.1080 / 13803390601186684$

Levine, B., Robertson, I. H., Clare, L., Carter, G., Hong, J., Wilson, B. A., et al. (2000). Rehabilitation of executive functioning: an experimental-clinical validation of goal management training. J. Int. Neuropsychol. Soc. 6, 299-312. doi: 10.1017/S1355617700633052

Martin, E. M., Pitrak, D. L., Weddington, W., Rains, N. A., Nunnally, G., Nixon, H., et al. (2004). Cognitive impulsivity and HIV serostatus in substance dependent males. J. Int. Neuropsychol. Soc. 10, 931-938. doi: 10.1017/S1355617704107054

Martin, E. M., Sullivan, T. S., Reed, R. A., Fletcher, T. A., Pitrak, D. L., Weddington, W., et al. (2001). Auditory working memory in HIV-1 infection. J. Int. Neuropsychol. Soc. 7, 20-26. doi: 10.1017/S1355617701711022

O’Brien, K. K., Ibáñez-Carrasco, F., Solomon, P., Harding, R., Cattaneo, J., Chegwidden, W., et al. (2014). Advancing research and practice in HIV andrehabilitation: a framework of research priorities in HIV, disability and rehabilitation. BMC Infect. Dis. 14:724. doi: 10.1186/s12879-014-0724-8

Orsini, A., Grossi, D., Capitani, E., Laiacona, M., Papagno, C., and Vallar, G. (1987). Verbal and spatial immediate memory span: normative data from 1355 adults and 1112 children. Ital. J. Neurol. Sci. 8, 539-548. doi: 10.1007/BF02333660

Orsini, A., and Laicardi, C. (1997). WAIS-R Contributo Alla Taratura Italiana. Firenze: Giunti OS.

Ownsworth, T. L., McFarland, K., and Young, R. M. (2002). The investigation of factors underlying deficits in self-awareness and self-regulation. Brain Inj. 16, 291-309. doi: 10.1080/02699050110103986

Robertson, I. H., and Murre, J. M. (1999). Rehabilitation of brain damage: brain plasticity and principles of guided recovery. Psychol. Bull. 125, 544-575. doi: 10.1037/0033-2909.125.5.544

Robertson, K., Liner, J., and Meeker, R. B. (2012). Antiretroviral neurotoxicity. J. Neurovirol. 18, 388-399. doi: 10.1007/s13365-012-0120-3

Rusch, M., Nixon, S., Schilder, A., Braitstein, P., Chan, K., and Hogg, R. S. (2004). Impairments, activity limitations and participation restrictions: prevalence and associations among persons living with HIV/AIDS in British Columbia. Health Qual. Life Outcomes 2:46. doi: 10.1186/1477-7525-2-46
Sacktor, N. C., Wong, M., Nakasujja, N., Skolasky, R. L., Selnes, O. A., Musisi, S., et al. (2005). The international HIV dementia scale: a new rapid screening test for HIV dementia. AIDS 19, 1367-1374.

Simioni, S., Cavassini, M., Annoni, J. M., Rimbault Abraham, A., Bourquin, I., Schiffer, V., et al. (2010). Cognitive dysfunction in HIV patients despite longstanding suppression of viremia. AIDS 24, 1243-1250. doi: 10.1097/QAD.0b013 e3283354a7b

Spinnler, H., and Tognoni, G. (1987). Standardizzazione e taratura italiana di test neuropsicologici. Ital. J. Neurol. Sci. 8(Suppl.), 1-120.

Stuifbergen, A. K., Becker, H., Perez, F., Morison, J., Kullberg, V., and Todd, A. (2012). A randomized controlled trial of a cognitive rehabilitation intervention for persons with multiple sclerosis. Clin. Rehabil. 26, 882-893. doi: $10.1177 / 0269215511434997$

Svendsen, H. A., and Teasdale, T. W. (2006). The influence of neuropsychologic rehabilitation on symptomatology and quality of life following brain injury: a controlled long-term follow-up. Brain Inj. 20, 1295-1306. doi: 10.1080/02699050601082123

Tesar, N., Bandion, K., and Baumhackl, U. (2005). Efficacy of a neuropsychological training programme for patients with multiple sclerosis - a randomised controlled trial. Wien. Klin. Wochenschr. 117, 747-754. doi: 10.1007/s00508005-0470-4

Thompson, P. M., Dutton, R. A., Hayashi, K. M., Toga, A. W., Lopez, O. L., Aizenstein, H. J., et al. (2005). Thinning of the cerebral cortex visualized in HIV/AIDS reflects CD4+ T lymphocyte decline. Proc. Natl. Acad. Sci. U.S.A. 102, 15647-15652. doi: 10.1073/pnas.0502548102

Underwood, J., Robertson, K. R., and Winston, A. (2015). Could antiretroviral neurotoxicity play a role in the pathogenesis of cognitive impairment in treated HIV disease? AIDS 29, 253-261. doi: 10.1097/QAD.0000000000000538

Vance, D. E., Fazeli, P. L., Ross, L. A., Wadley, V. G., and Ball, K. K. (2012). Speed of processing training with middle-age and older adults with HIV: a pilot study. J. Assoc. Nurses AIDS Care 23, 500-510. doi: 10.1016/j.jana.2012.01.005

van Gorp, W. G., Rabkin, J. G., Ferrando, S. J., Mintz, J., Ryan, E., Borkowski, T., et al. (2007). Neuropsychiatric predictors of return to work in HIV/AIDS. J. Int. Neuropsychol. Soc. 13, 80-89. doi: 10.1017/S1355617707070117

Weber, E., Blackstone, K., and Woods, S. P. (2013). Cognitive neurorehabilitation of HIV-associated neurocognitive disorders: a qualitative review and call to action. Neuropsychol. Rev. 23, 81-98. doi: 10.1007/s11065-013-9225-6

Wood, R. L., and Rutterford, N. A. (2006). Psychosocial adjustment 17 years after severe brain injury. J. Neurol. Neurosurg. Psychiatr. 77, 71-73. doi: 10.1136/jnnp.2005.065540

World Medical Association (2013). Declaration of helsinki: ethical principles for medical research involving human subjects. JAMA 310, 2191-2194. doi: 10.1001/jama.2013.281053

Worthington, C., Myers, T., O’Brien, K., Nixon, S., and Cockerill, R. (2005). Rehabilitation in HIV/AIDS: development of an expanded conceptual framework. AIDS Patient Care STDS 19, 258-271. doi: 10.1089/apc.2005. 19.258

Conflict of Interest Statement: The authors declare that the research was conducted in the absence of any commercial or financial relationships that could be construed as a potential conflict of interest.

Copyright (c) 2015 Livelli, Orofino, Calcagno, Farenga, Penoncelli, Guastavigna, Carosella, Caramello and Pia. This is an open-access article distributed under the terms of the Creative Commons Attribution License (CC BY). The use, distribution or reproduction in other forums is permitted, provided the original author(s) or licensor are credited and that the original publication in this journal is cited, in accordance with accepted academic practice. No use, distribution or reproduction is permitted which does not comply with these terms. 